\title{
Field geologic observation and sample collection strategies for planetary surface exploration: Insights from the 2010 Desert RATS geologist crewmembers
}

\author{
José M. Hurtado Jr. ${ }^{a, *}$, Kelsey Young ${ }^{b}$, Jacob E. Bleacher ${ }^{c}$, W. Brent Garry ${ }^{d}$, James W. Rice Jr. \\ ${ }^{a}$ University of Texas at El Paso, Department of Geological Sciences, 500 West University Avenue, El Paso, TX 79968-05555, United States \\ ${ }^{\mathrm{b}}$ School of Earth and Space Exploration, Arizona State University, Bateman Physical Sciences Center, F-wing, Room 686, Tempe, AZ 85287-1404, United States \\ ${ }^{\mathrm{c}}$ Planetary Geodynamics Laboratory, Code 698, National Aeronautics and Space Administration, Goddard Space Flight Center, 8800 Greenbelt Rd., Greenbelt, \\ MD 20771, United States \\ d Planetary Science Institute, 1700 East Fort Lowell, Suite 106, Tucson, AZ 85719, United States \\ e Solar System Exploration Division, Planetary Geodynamics Laboratory, Code 698, NASA Goddard Space Flight Center, Greenbelt, MD, United States
}

\section{A R T I C L E I N F O}

\section{Article history:}

Received 1 July 2011

Received in revised form

6 October 2011

Accepted 24 October 2011

\section{Keywords:}

Field geology

Planetary analog

Sample collection

Field geology methods

Extravehicular activity (EVA)

Planetary field geology

\begin{abstract}
A B S T R A C T
Observation is the primary role of all field geologists, and geologic observations put into an evolving conceptual context will be the most important data stream that will be relayed to Earth during a planetary exploration mission. Sample collection is also an important planetary field activity, and its success is closely tied to the quality of contextual observations. To test protocols for doing effective planetary geologic fieldwork, the Desert RATS (Research and Technology Studies) project deployed two prototype rovers for two weeks of simulated exploratory traverses in the San Francisco volcanic field of northern Arizona. The authors of this paper represent the geologist crewmembers who participated in the 2010 field test. We document the procedures adopted for Desert RATS 2010 and report on our experiences regarding these protocols. Careful consideration must be made of various issues that impact the interplay between field geologic observations and sample collection, including time management; strategies related to duplication of samples and observations; logistical constraints on the volume and mass of samples and the volume/transfer of data collected; and paradigms for evaluation of mission success. We find that the 2010 field protocols brought to light important aspects of each of these issues, and we recommend best practices and modifications to training and operational protocols to address them. Underlying our recommendations is the recognition that the capacity of the crew to "flexibly execute" their activities is paramount. Careful design of mission parameters, especially field geologic protocols, is critical for enabling the crews to successfully meet their science objectives.
\end{abstract}

(c) 2011 Elsevier Ltd. All rights reserved.

Abbreviations and definitions: 2/D, twice-per-day communications; CC, continuous communications; CFN, crew field note; Cuff, arm-mounted computer control for CFN system; D\&C, divide-and-conquer; Desert RATS, Desert Research and Technology Studies; Drive, movement of SEV between Stations; EVA, extravehicular activities; Flexecution, flexible execution; GigaPan, robotic pan-tilt-zoom camera mount for high-resolution panoramic imaging; GIS, geographic information system; High-grading, prioritization for potential Earth-return; IVA, intravehicular activities; KML-format, Google Earth file format for GIS data; L\&F, lead-and-follow; Science Backroom, subset of the Science Team that supports the crew during CC; Science Team, mission control personnel that support, guide, and direct the science operations done by the crew; SEV, space exploration vehicle; Station, location where SEV has stopped for science or operational work (with or without an EVA); Suitport, rear hatches on the SEV that allow quick egress/ingress for EVAs along with dust mitigation; Traverse, series of Stations and drives

* Corresponding author. Tel.: +1 915747 5669; fax: +1 9157475073.

E-mail address: jhurtado@utep.edu (J.M. Hurtado Jr.). 


\section{Introduction}

Desert Research and Technology Studies (Desert RATS) is a multi-year series of hardware and operations tests conducted annually since 1997 in the high desert of Arizona [1, this issue]. Integrated teams of engineers and scientists have merged exploration technologies with exploration strategies to support these ambitious field tests, which allow testing of science operations approaches that advance human and robotic planetary surface exploration capabilities. The centerpiece of these tests is a pair of crewed rovers designed as first-generation prototypes of small, pressurized space exploration vehicles (SEVs) [1]. The SEV provides the internal volume necessary for two crewmembers to live and work nearly-autonomously on simulated planetary missions for up to 14 days $[1,2]$. Investigations can be conducted from within the pressurized environment of the SEV (i.e. IVAs, or intravehicular activities) using a suite of cameras and other sensors mounted on the SEV [3]. The crew can also conduct extravehicular activities (EVAs) for geologic fieldwork through the use of rear-mounted "suitport" hatches that allow quick egress and ingress, greatly increasing the flexibility and efficiency of EVAs while enabling the crew to recover and prepare for the next EVA and make observations in the shirtsleeve environment of the SEV [2,4].

Desert RATS 2010 took place between 31 August and 13 September 2010 and included two pairs of crews, each consisting of an engineer/commander and an experienced field geologist. Three of the engineer/commanders were experienced astronauts with at least one Space Shuttle flight, and the four field geologists were drawn from the scientific community (both academia and NASA). Two crewmembers lived in, drove, and worked from each SEV for a single week, with a "shift change" on day 7, resulting in a total of eight test subjects for the two week period. The field test was designed to execute geologic traverses through a terrain of cinder cones, lava flows and underlying sedimentary units in the San Francisco volcanic field north of Flagstaff, Arizona. Prior to the test, a series of traverses were planned in conjunction with the U.S. Geologic Survey Astrogeology Branch using techniques that were first developed during Apollo [5,6]. The traverses were designed to simulate a reconnaissance investigation of a planetary surface with a variety of communications and operational constraints. For example, three days of each week were tested with the SEVs in continuous communications (CC) with mission operations team, and three days were tested with communications only for $\sim 1 \mathrm{~h}$ in the morning and $\sim 1 \mathrm{~h}$ at the end of the traverse day (2/D). Similarly, the SEVs were tested in two operational modes: lead-andfollow (L\&F) and divide-and-conquer (D\&C). See [1] in this issue for a detailed discussion of the Desert RATS series of tests, including the 2010 test, and refer to [7] in this issue for a discussion of the crew perspectives on the effect of these operational and communications modalities on science capabilities.

From a science operations perspective, the main objective of Desert RATS has been to examine the functions of a science support team, the roles of geologist crewmembers, and the protocols, tools, and technologies needed for effective data collection, data transfer, and curation of samples. The latter is a particularly important, difficult, and multifaceted problem, and its solution must consider how traditional, terrestrial modes of field geology will need to be adapted to geologic field work in extreme environments [8], particularly during EVAs. Careful consideration must be made of various issues that impact the interplay between field geologic observations and sample collection. These include the time spent making observations versus collecting samples; strategies related to the number, type, and degree of duplication in samples and observations; logistical constraints on the volume and mass of samples that can be collected and ultimately returned to Earth and the volume of data that can be recorded and transmitted to Earth; and paradigms for evaluation of mission success. This contribution reports on the Desert RATS geologist crew experiences regarding these issues. We document the procedures adopted for Desert RATS 2010 and the crew perspectives on this aspect of field science operations, describe our lessons learned, and offer recommendations for future analog tests and planetary missions.

\section{Field geology in a planetary exploration context}

Among the primary science motivations for planetary exploration is to decipher the development of the solar system, a history that is documented in the geologic records of planetary bodies. The geological sciences, therefore, lay the foundation for fundamental questions regarding the chemical, biological, and geodynamic evolution of planets, and, because of this, expertise in geology will be a critical skill set for crewed planetary exploration missions [9]. Previous Desert RATS field tests have demonstrated the value of including trained field geologists on planetary exploration crews $[2,4,10]$, as was also the case with Schmitt on Apollo 17 [9].

Observation is the primary role of all field geologists, including those on a planetary exploration crew. Geologic observations reported verbally and supported by imagery, and the evolving conceptual context built around them, are the most important data stream that will be relayed to Earth. It is through these observations and interpretations that the geologic context and history of a field site will be documented and investigated. Field observations also support sample collection, a necessary, but usually secondary, task in field geology. While samples are vital for quantitative chemical, isotopic, and other analyses, the quality of those samples, and of the resulting datasets, is highly dependent on the observations made in the field. Most importantly, those observations provide the vital context required to meaningfully interpret the samples, the data derived from them, and their relationship to other samples and to geologic processes. Therefore, it is important to realize that field observations contribute information that samples alone would not. For example, a given suite of samples without context cannot inform the geologist about the fundamental geometric and structural relationships traditionally captured on a geologic map. For these reasons, terrestrial field geologists have long appreciated the complementary balance between observation and sample collection for understanding the geologic history of a site, as well as the processes behind it. Therefore, the way in which observational information is documented, organized, 
transferred, displayed, and integrated with all other data is of the utmost importance for planetary exploration.

To date, planetary exploration by humans has been focused heavily on sample collection and with great success. For example, the six Apollo missions to the moon returned $382 \mathrm{~kg}$ of material [11] from which fundamental scientific discoveries are still being made. To accomplish their fieldwork, the Apollo astronauts followed highly choreographed field protocols during tightly scheduled EVAs that were generally scheduled to the minute [11]. These activities were necessarily focused on directed, contextual sampling, rather than local- to regional-scale observations, a situation driven primarily by time limitations imposed by EVA consumables and other mission logistical considerations. Nevertheless, several of the Apollo crews, who almost entirely comprised non-geologists, distinguished themselves with the observations they made. For example, David Scott and Jim Irwin on Apollo 15 insisted on an innovative "stand-up" EVA prior to their three surface traverses [11,12]. The stand-up EVA, conducted by Scott while standing inside the lunar module with his head outside the top hatch, established local and regional context using photography and verbal descriptions of the Hadley Apennine landing site. The voice transcripts of these descriptions and the accompanying photographs (e.g. Apollo Lunar Surface Journal; [11]) have proved invaluable for interpreting what was seen and collected at the EVA Stations visited later on in the mission.

Future human exploration of planetary surface will advance beyond what was achieved by Apollo with new approaches that place as much emphasis on observation as on sampling. The strategies for making science observations and collecting samples in these missions must also embrace the notion of "flexecution", or flexible execution $[9,13,14]$. In this way, hypothesis-driven science objectives are clearly defined, but they are also subject to continual modification, as is the timeline during which the field activities designed to meet those objectives are conducted. This is a fundamentally different approach from Apollo. As described by Hodges and Schmitt [8], flexecution is the standard concept of operation for terrestrial field geologists and should serve as the model for how planetary field geology is done. Flexecution powerfully enables the experienced field geologist to adapt her conceptual model of the science simultaneously with her operational plan for how to collect scientific data in real time as new observational data is collected. At the least, the result is a traverse specifically and efficiently focused on the problem at hand, and, ideally, this leads to enhanced scientific understanding and the ability to adapt to unanticipated discoveries and other scientific and operational opportunities and challenges.

Recognizing that many field science discoveries are the product of serendipity - often because traverses designed from remotely sensed data lack local contextual information from the ground, and, as such, cannot be planned flexecution allows the terrestrial field geologist to effectively exploit scientific targets of opportunity as they arise. Traverse planning and reconnaissance can never capture the full spectrum of possibilities, so having the ability to positively react to the unanticipated is crucial for planetary field geology as well. Interestingly, this is an exploration philosophy at the heart of some robotic exploration missions such as the Mars Exploration Rovers [15]. Even with their strict timelines, the Apollo astronauts were able to profitably improvise at times. For example, Scott and Irwin during EVA 1 on Apollo 15 surreptitiously collected the "seatbelt basalt" (sample 15415) at the unplanned Station 3 [11,12]. Scott's geologic training allowed him to realize the potential interest this sample held, and only an immediate, on-the-scene decision to stop allowed that sample to be collected, which was fortunate because that sample proved to be one of the best examples of vesicular mare basalt collected during Apollo. Science operations protocols for the future must recognize the need for such flexibility and therefore reasonably allow for improvisation within the constraints of the mission.

Even when done opportunistically, sample collection is important, but only if done carefully and judiciously. For example, some samples obtained by Alan Bean at the end of EVA 2 on Apollo 12 were semi-randomly collected without sample bags or contextual photography $[11,16]$. This has limited their usefulness because they cannot be reliably placed into specific, site-level geologic context. It is also important to ensure that collecting opportunistic samples, which can be done occasionally, does not come at the cost of not collecting "average" samples that are more representative of the geology of a site. Worthwhile sampling takes careful consideration of various other issues, including well developed, testable hypotheses; proper and complete characterization of context; a balance between time spent sampling versus observing; degree of redundancy; volume and mass limitations; and the capacity of "high-grading" (prioritizing) samples at mission's end. Most important, though, is the notion that sample collection must be done with a purpose, i.e. to test well developed hypotheses and to serve as a reference for future measurements and comparison to other field sites. Otherwise, the collected samples become a very expensive hobby rock collection, rather than a contributor to a greater scientific understanding. In the following sections we describe how each of these issues were approached for Desert RATS 2010 and the insights the crews gained.

\section{Desert RATS 2010 field geology protocols}

The traverse planning process for Desert RATS 2010 is described in detail by Horz et al. [5] and Skinner and Fortezzo [6], both in this issue, and resulted in the preselection of EVA Stations as well as definition of science priorities at these Stations and the drives in between (for definitions of the terms traverse, drive, Station, and EVA used here, see also [7] in this issue). These plans were then interpreted and executed in real time by the crews in concert with their Science Backrooms (the subset of the Science Team that supported the crew during CC, for details, see $[7,17]$ in this issue). Since the crewmembers have de-facto final authority for science activities, it is critical that the crews be well briefed on the science objectives and operational modes [7] and well trained in the use of the provided tools [18] and the expected field procedures. In this section, we describe the Desert RATS 2010 EVA field procedures as executed by the crews 


\section{Desert RATS 2010 Field Geology Protocols}
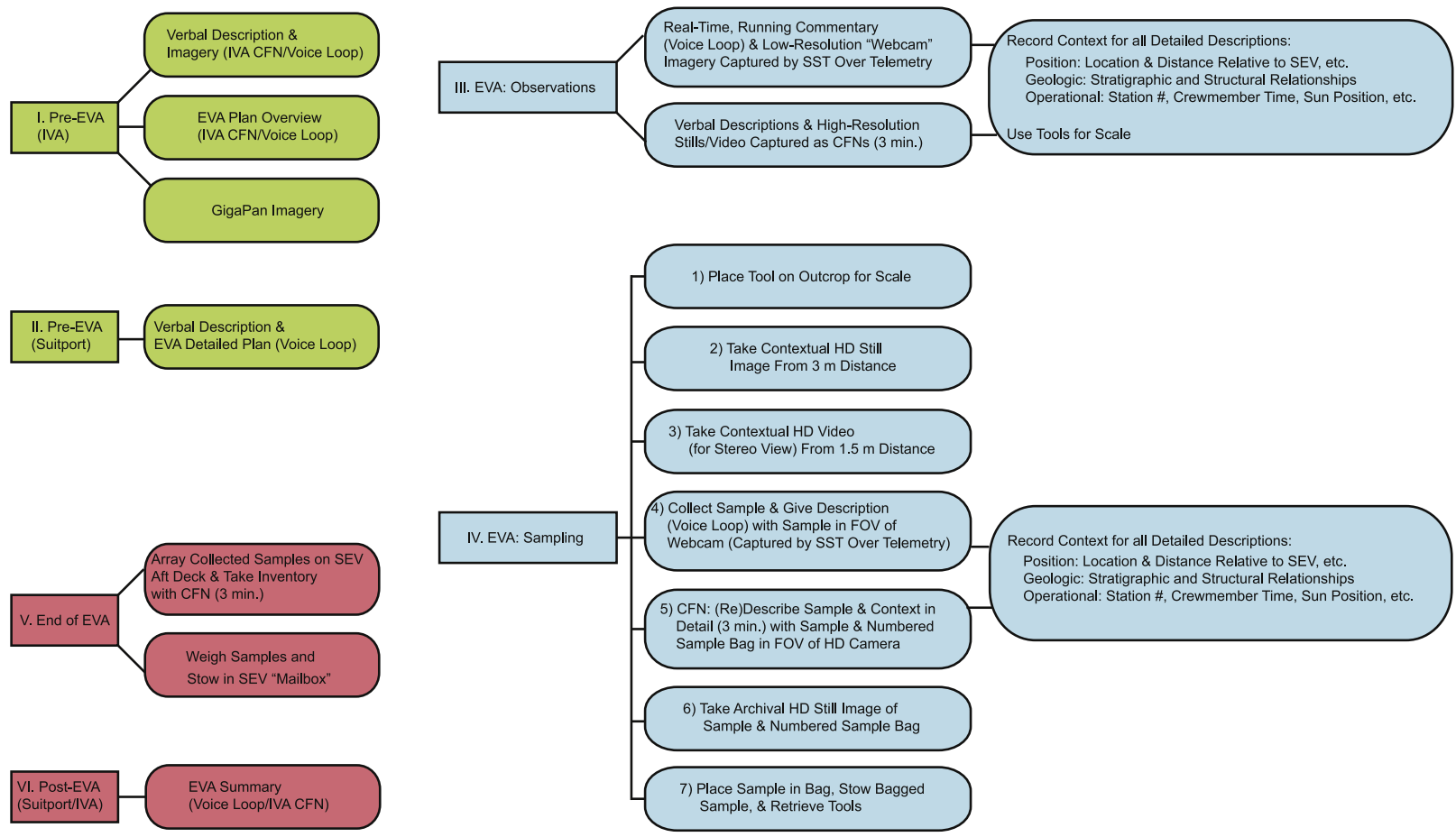

Place Sample in Bag, Stow Bagged

Fig. 1. Flowcharts summarizing the field geology protocols followed during the 2010 Desert RATS field test. Sections 1 and 2 denote activities carried out prior to EVAs. Sections 3 and 4 enumerate the procedures followed for geologic observations and for sample collection during EVAs, respectively. Sections 5 and 6 show the activities done at the end of an EVA.

(Fig. 1). These were designed from the perspective of capturing as much contextual data as possible for the proper curation of collected samples. Similar protocols were followed to document generic field observations. The entire process of conducting field geology during EVAs is heavily technology-dependent, including communications/telemetry and tools. Young et al. [18] in this issue give more details about the crews' experiences with tools while on EVA.

\subsection{IVA procedures for geologic observations and EVA planning}

EVA operations begin during the drive towards a Station, during which time the geologist reviews the site objectives and develops a preliminary plan for the EVA based on what can be seen first hand from the SEV. During CC operations, this is done over the voice loop in collaboration with the Science Backroom, but during 2/D operations the geologists on each SEV work together to develop their plans [7], documenting the process using IVA Crew Field Notes (CFNs; see also $[18,19]$ in this issue). Whether on CC or 2/D, the geologist also records an IVA CFN documenting the geology of the Station and their EVA plan prior to arrival.

Once on Station, and prior to egress, the geologist initiates a GigaPan - a high-resolution, up to $360^{\circ}$ panoramic photomosaic obtained using a robotic camera mounted on the SEV [20] - that includes the planned EVA site(s). This is done to ensure that high-resolution photography is captured of the entire area to be visited by the crew so that samples and observations can be put in spatial context. In this way, the GigaPan is analogous to the photographic base for an outcrop map. After GigaPan image acquisition has been initiated, the crew makes final preparations to egress, first ensuring that the GigaPan is successfully acquiring (a consideration during 2/D communications when the Science Backroom could not intervene) and that all SEV cameras are switched to Science Backroom control. In this way, the Science Backroom can track the crew as they work using the live video feeds. Similarly, the Science Backroom (during $\mathrm{CC}$ ) has the ability to capture additional GigaPan mosaics documenting the progress of the EVA activities.

Finally, while the crewmembers are on the suitports at the rear of the SEV finishing the egress procedures, the geologist and astronaut do a pre-briefing of the EVA plan with each other and make further observations of the geologic context of the Station. This conversation between crewmembers is not captured in a CFN, but is done over the voice loop so that the Science Backroom can participate when CC is available. Pre-positioning of the SEV before egress is important in this respect as ideally the suitports are pointed in the direction of the outcrop so that observations can be made while the crew is waiting for the pre-EVA leak checks to complete.

These pre-EVA activities done from within the SEV (or from the suitport) are critical steps as they are the opportunity for the crew to characterize the site at a large scale, to 
detail their plan of action, and to establish the context for their geologic fieldwork, including samples to be collected and their locations. Making as many observations as possible before embarking on the EVA saves precious time and consumables and results in an initial plan for the EVA that ensures the fieldwork is focused and efficient.

\subsection{EVA procedures for geologic observations}

Terrestrial geologists have the luxuries of time and mobility while doing fieldwork that are not entirely shared by a planetary field geologist/astronaut. Once the EVA has started, there is a finite and relatively short amount of time at the Station for accomplishing the objectives, which is why the time spent pre-planning (see Section 3.1) is critical for ensuring efficiency and success. Similarly, the procedures and tools for recording observations and for collecting samples (see Section 3.3) are designed with the limitations of planetary fieldwork (e.g. writing in a field notebook is not possible in a spacesuit) and the constraints of the EVA timeline in mind [18].

Both SEV crewmembers are expected to maintain a running commentary of their observations and other nonsampling activities throughout the EVA during both CC and 2/D scenarios in order to keep both the Science Backroom and their partner apprised of their progress. Since the crewmembers share a single voice loop while on EVA, it is important that they coordinate so that they do not interfere with each other's verbal communication and commentary. During CC, but not during 2/D, the running commentary done over the voice loop is interpreted and recorded/transcribed in real-time by the Science Backroom. This commentary is accompanied by a low-resolution live video feed captured by one of the two cameras on the simulated spacesuit backpacks that the crewmembers wear [18]. During both CC and 2/D, the crewmembers also have the option of capturing $\leq 3-$ min long EVA CFNs intended for high-priority download and analysis by the Science Team at the end of the mission day. The EVA CFNs are recorded using the second, high-definition, camera on the crew backpacks, along with a cuff-computer-controlled voice annotation system [18].

\subsection{EVA procedures for sample collection}

While on EVA, each sample is collected in a particular manner using a variety of tools, including a rock hammer, shovel, tongs, core-tube, and Teflon sample bags, although it is important to note that the crew lacked a hand lens, one of the most basic tools used by a field geologist (for details see [18] in this issue). As with geologic observations, each sample is documented using both the real-time video/audio telemetry (particularly during $\mathrm{CC}$ ) as well as with an EVA CFN video clip (during both CC and 2/D). The procedural checklist for collecting samples is enumerated in Fig. 1.

In the case of both sample documentation and standalone geologic observations, contextual details are critical and each crew devised their own semi-formal checklist of items to note (see Fig. 1 for examples). In addition to the requisite geologic data about the stratigraphic and structural relationships observed in the field, this checklist includes spatial and operational metadata for context. These allow simple placement of the geologic information within the framework of the EVA timeline and spatial extent so that, for example, samples could be referenced to the context imagery taken from the SEV (e.g. the GigaPans) and the CFNs could be interpreted in a chronologic order.

\subsection{Procedures for sample stowage and EVA closeout}

At the end of the EVA, a complete inventory is taken before stowing the samples on the SEV. With all the bagged samples arrayed on the aft deck of the SEV, so that the sample numbers are visible, an EVA CFN listing all the samples is recorded. This was an informal procedure that was initiated by the week $1 \mathrm{crew}$ and soon made a standard part of the EVA for all crews. Once the CFN is complete, the bagged samples are gathered into a daily collection bag, which is then weighed and stowed in a storage receptacle on the SEV aft deck [18]. These sample inventories are useful for locating samples at the end of the mission and also for locating samples for "highgrading" and/or in-situ analysis in the surface habitat [21]. They have also proved valuable for reconstructing the samples collected at Stations for which all other documentation was lost due to telemetry, communications, or other data transfer problems [22].

Similarly, a verbal overview of the geologic observations made by both crewmembers during the EVA and hypotheses on how those observations fit into the larger geologic picture is recorded at the end of the EVA. The exact way this was done varied from geologist-to-geologist during Desert RATS 2010. Every crewmember recorded an EVA CFN either before or after the sample inventory, as time allowed, usually followed by a detailed summary as an IVA CFN once the crew had ingressed the SEV. In addition, most of the crew also recorded these overviews in text and spreadsheet documents on the onboard computer in the SEV, often during drives between Stations. It should be pointed out, however, that these data files were underutilized since the Science Team was not always aware of where they were stored (see Section 4.3; $[7,18]$ in this issue).

\subsection{Improvised IVA and EVA field procedures}

Over the course of the 2010 field test, other procedures related to sample and data collection that differed from the protocol lists given to the crew for the mission were improvised and adopted. Two of these adapted procedures are described here.

\subsection{1. “Grab-bag" sampling}

All of the crewmembers eventually adopted a bag clipped to their waists for carrying bagged samples while on EVA instead of the supplied tool/sample caddy [18]. As a consequence, they also had a convenient receptacle in which to put "grab" samples. These were rocks, most often found in float, that, for one reason or another looked visually interesting and were picked up opportunistically. Because they were picked up at odd times during the EVA - e.g. while on a long walking traverse or at the very 
end of the EVA in the hurry back to the SEV - these samples were typically not bagged or documented following the standard protocol (Section 3.3), although the crews did make efforts to document them as time allowed, which was typically a hasty CFN recorded while walking. No specific and consistent procedure was developed for this style of sample collection, however.

\subsubsection{Geologic mapping}

Many of the SEV systems are dual-purpose [3]. For example, the onboard cameras are used for science observations by both the crew and the Science Team, but also for mission operations purposes such as navigation and hazard avoidance. Similarly, the Google Earth navigation system is used for driving the SEV as well as for displaying maps showing communications coverage and the preplanned traverse routes.

The week 2 crewmembers made additional use of the camera and navigation systems for rudimentary geologic mapping. For example, to supplement the voice and camera imagery included in the end-of-EVA CFNs, the GigaPan mosaics were annotated using the available "snapshot" tools [20] to graphically illustrate the route taken on the outcrop(s) and the exact location of collected samples. These rudimentary outcrop maps were produced at most stations during week 2 and were well received by the Science Team as a tangible increase in productivity as compared to during week 1 (when the crewmembers were not aware of this electronic capability). At another Station, an effort was also made, using Google Earth as a rudimentary GIS (geographic information system), to produce a KML-format overlay showing the geologic contacts and a suspected fault that were observed, essentially a simple geologic map.

\section{Crew insights and lessons learned}

Here we discuss some insights gained by the crewmembers into best practices for planetary fieldwork that were the result of performing the geologic observation and sample collection tasks during the 2010 field-test. It is important to note that these depend strongly on the duration and goal of the mission. Desert RATS has simulated 7-14-day missions on destinations such as the Moon. Our lessons learned and the recommendations (Section 5) that stem from them are valid within that framework. However, for longer mission durations and for other destinations, such as a near-earth asteroid, the results could be different.

\subsection{Time management on EVA}

Given the protocols described in Section 3, it takes approximately $10 \mathrm{~min}$ to collect each rock sample. This is a relatively large time resource commitment, given that most EVAs during Desert RATS 2010 were between 30 and $60 \mathrm{~min}$ in length. Moreover, a significant percentage of these $10 \mathrm{~min}$ are committed prior to picking up the rock (i.e. taking context imagery, etc.). This is particularly challenging if there are multiple geologic units and contacts to describe and sample at a given EVA Station. There may also be a lot of ground to cover at a spatially extensive or topographically rugged EVA Station. Therefore, time management on EVA is a critical consideration. The crew must continuously balance the time used to collect multiple samples versus the time to best characterize an outcrop and, as a result, perhaps collect fewer, yet higher-quality, samples. In addition, if the crew chooses to spend more time at a particularly interesting site, they must also sacrifice time somewhere else in that day's traverse. These decisions to focus on observation or sample collection at any given EVA Station are made in real-time based on the science objectives and the first-hand observations of the crewmembers. These decisions are interdependent on other considerations, including duplication of effort (Section 4.2), the amount and types of samples and observations obtained (Section 4.3), and the use of tools/technologies [18].

The decision of when to collect a sample and when not to can also affect the way mission success is measured from a science perspective (Section 4.4). In terms of mission success, the value of geologic observations must be recognized as (at least) equal and complementary to the value of tangible geologic specimens. This is only the case, however, when there is an appropriate balance between sampling and observation, as the quality of the former is directly dependent on the latter. In this context, there is a distinction to be made between poor-quality samples and samples collected poorly. A poor-quality sample is one that is, for example, lithologically unsuitable for the purposes intended or the question to be answered (e.g. it is weathered versus fresh, it is the incorrect unit, etc.). A poorly-collected sample means that time was mismanaged so that more samples were collected at the expense of not documenting them correctly, rushing to collect rocks before fully understanding their context. Both are to be avoided and the way to do so is to include well-trained field geologists on a planetary exploration crew and for them to take the time to carefully document a site before sampling. The reverse is also true, particularly given the time and logistical constraints imposed on the geologist during an EVA. Because it is also important for the mission that samples be collected, the planetary field geologist must be enabled to be decisive, make concise and cogent observations, and work in a well-informed and purposeful manner. Overall, doing fieldwork without purpose is a problem, which is why analog tests like Desert RATS are important for helping train crews to see the purpose behind what they do in the field, both scientifically and operationally.

Mission plans, field protocols, and tools should be designed to properly enable decision-making to support an effective balance between geologic observations made and samples collected. For Desert RATS 2010, one operational strategy we found that worked was to create a sampling plan during the IVA period and prior to the boots-on-the-ground EVA when in the suitport (see protocols described in Section 3). Having a preliminary strategy in place before EVA minimized searching the outcrop while on EVA, maximizing the balance between observations and samples, and it also prepared us for making real time decisions and changes to the plan as the EVA progressed (i.e. flexecution). Another strategy the crews experimented with was to either work together or split up on EVA. For example, sometimes we chose to stay close together, with 
one crewmember taking context imagery while the other described the samples [19]. At other times, we split up, with both crewmembers traveling relatively far from the SEV and each other (in accordance with flight rules) to cover more ground [19]. Even with these strategies, we found room for improvement in the sample documentation protocols. In addition, while meticulously recording geologic context and carefully isolating a sample are critical, they are timeconsuming procedures. We offer recommendations regarding these issues in Section 5.

\subsection{Duplication of effort}

For missions where multiple rovers and crewmembers conduct geologic fieldwork on EVA, duplication of effort may occur with respect to observations made and samples collected. This could negatively affect the use of the precious resources of time, bandwidth, data storage, and physical stowage space (e.g. volume and mass allowances) (see Section 4.3). While we do not deny that minimizing unnecessary duplication is worthwhile, we can identify ways of mitigating it and even use it as an advantage.

At first, it may seem that duplication of effort could be used as a measure of crew effectiveness in analog tests (see Section 4.4), as it might suggest poor traverse planning or the need for more coordination among the crews. However, we argue that this is not necessarily a good metric to use in either a training/simulation or real mission scenario. For example, a more important cause of potential duplication of effort during Desert RATS 2010 was the communications architecture. Given the communications structure for EVAs, it was impossible for the two SEV crews on EVA to know what the other one was doing once they were out of their vehicles: one crew could not talk to their counterparts on the other crew over the voice loop (only face-to-face communication was possible and only when the crews were in very close proximity). Since on 2/D-L\&F days the SEVs worked in the same general area, it was likely that the same units would be sampled and similar observations would be made.

In the context of the 2010 field test, the available data storage and bandwidth on the SEV proved sufficient to handle the volume of observations and associated digital data. We point out, though, that the only instruments we used were cameras, so this assessment does not consider the effects of future incorporation of high-bandwidth tools such as LiDAR (light detection and ranging), visible-infrared spectrometers, or geochemical instruments. However, given the infrastructure concepts that were tested, we found that duplication of observational data did not pose a serious data management problem, although telemetering of that data was fraught with other difficulties (see Section 4.3; $[7,18]$ in this issue). In contrast, storage of samples was somewhat logistically problematic in terms of the mass and volume constraints of the SEV (see Section 4.3). Part way through both week 1 and week 2 , additional sample bags had to be supplied to the crew and samples had to be offloaded from the SEV to make room for additional ones, operations that were conducted outside of the simulation. For a real mission that may operate on stricter and tighter logistical margins, this suggests that any duplication of samples may be a significant problem to manage unless the storage constraints of the spacecraft are augmented or procedures such as sample caching or in-situ "high-grading" [21] are implemented. In addition, using assets such as the Pressurized Excursion Module (PEM)/GeoLab [21] for in-situ analyses and/or robotic follow-up [23], "excess" samples that cannot be returned to Earth can still be of scientific value, particularly if sufficiently documented upon their collection, so that the resources used to obtain them did not go to waste.

The detrimental effect duplication has on time and science return remains another important issue. Unnecessary and excessive duplication of fieldwork wastes EVA time, with potentially little benefit to science outcomes. Alternatively, a limited degree of duplication may in fact be tolerable, if not beneficial. For example, some duplication may retire the scientific risk of losing critical samples in the unfortunate event of some sort of mission failure or to ensure that material remains for vital laboratory work. Although not done during Desert RATS 2010, contingency samples for this purpose were collected by each Apollo mission at their landing sites as the first task upon egressing from the lunar module [11]. A situation where duplication retired scientific risk during Desert RATS 2010 stemmed from the inter-crew communication limitation experienced during 2/D-L\&F EVAs. Since, without continual coordination, duplication of effort was inevitable, the conservative course of action was to comprehensively sample. The understanding is that it is better to have duplication than to have one crew assume the other has sampled a unit when, in fact, no one did.

Duplication of samples will certainly be of value, if not required, for some science objectives. For example, multiple specimens of the same geologic material at a number of scales may be needed to determine lateral or vertical variability not visibly apparent in the field (e.g. isotopic or geochemical trends). Similarly, some analytical methods (e.g. geochronology/thermochronology) often benefit from multiple, independent samples, either from the same place or from different places. Others may require replicate analyses for statistical purposes.

With respect to duplication of observational data collected, field geology has always enjoyed the benefit of multiple perspectives on problems. Having observational data about the same feature or unit from more than one crewmember can provide important insights into processes [7]. In fact, this mode of making complementary observations, and thereby feeding the collaborative process of developing multiple working hypotheses, is a cornerstone of geologic fieldwork.

\subsection{Storage considerations}

Each crewmember sampled approximately the same number/mass of samples, and none of the crewmembers took an excessive number of samples compared to what would be collected during terrestrial fieldwork [24]. However it is important to note that potential stowage constraints do exist as they relate to duplication of effort (see Section 4.2), and they will also be limiting factors on the individual sizes of samples collected (i.e. sample bag volume) and the total number of samples collected per 
EVA or per mission (i.e. volume of sample stowage containers on the rover) [18].

These constraints have implications for geologic fieldwork protocols and the selection and collection of samples. For example, with the $17.78 \times 22.86 \mathrm{~cm}^{2}$ (7-inch by 9 -inch) bags used during Desert RATS 2010, the crew had to ensure that the pieces of rock they collected fit in a single bag since the curatorial infrastructure did not easily support keeping track of samples stored in multiple bags. In addition, the sampling strategy in one field area may focus on the collection of rock and soil samples whereas other field sites, on simulated or real missions, may require different handling of other types of materials (e.g. liquids, biological, etc.). For example, Desert RATS incorporated some aspect of this in the form of the core tube samples [18] collected on at least one EVA per week, and several varieties of specialized sample containers (e.g. the Special Environmental Sample Container) were fielded on the Apollo missions [11]. Hence the sample bag/receptacle design directly puts limitations on the types and amounts of samples collected and the eventual use of those samples. This in, turn, has a direct effect on the procedures used to collect the materials. In the case of rock samples, the bag size also affects EVA time as breaking a sample of an appropriate size takes time, particularly if it is a piece liberated directly from solid outcrop. This, in turn, affects the bag design itself as the sharp edges on freshly broken surfaces can abrade or cut open sample bags, potentially leading to cross-contamination or loss of valuable material [18].

Future field tests and actual missions may include larger sample bags that allow for larger and more scientifically versatile samples [18], decisions that will be driven by the science community's minimum and maximum sample size requirements. Such (larger) samples may be used to illustrate particular, scale-dependent structures or textural features (e.g. ripple marks on sandstone) or to provide enough material for particular kinds of analyses, although it is important to keep in mind that such samples are often made superfluous if the appropriate geologic relationships are documented at the larger scale. To best use whatever storage capabilities are available, though, sampling procedures will need to be developed to guide the crew in choice of sample size given the intended use of the material. This will require that the crew be familiar with whatever PI-driven science requirements have shaped the EVA and traverse plans [7] and will require specialized training in the proper collection of samples intended for specialized purposes (e.g. oriented samples, environmentally-sensitive samples, etc.) or for sampling otherwise unexpected finds.

As described by Young et al. [18], stowage and transport of tools were also problematic and impacted geologic fieldwork protocols. A particular innovation to the sampling protocols that was facilitated by the resulting adoption of clipped-on bags was the "grab sample" (see Section 3.5.1). While the number of samples collected in this manner is small and had a negligible effect on timeline or space considerations, it remains to be seen what the scientific value of such materials is, especially when documentation and contextual information at times were sparse to nonexistent $[21,22]$. Nevertheless, the fact that some samples will always likely be obtained outside the established protocols (e.g. the Apollo 15 "seatbelt basalt"; Section 2; [12]) requires that flexibility be built-in to the documentation procedures. This has implications for technology, as the infrastructure for rapidly and reliably documenting and tagging samples must be there [18].

With regard to non-sample science products, e.g. observations, the crew experience with data volume (bandwidth, etc.) during fieldwork showed that there was ample capacity for handing the data files, although the inclusion of scientific instruments in the future will undoubtedly test the limits of the telemetry. On multiple occasions, procedural oversights hampered the optimal use of some of these data files since the Science Team was not always cognizant of the fact that these files, such as end-of-day Excel spreadsheets, were produced or where they were stored. This highlights the critical need for data collection procedures to specify not only what data is to be recorded but also where it is to be stored and how it is to be accessed, so that nothing is lost or left out. The other principal technological bottlenecks during Desert RATS 2010 arose from communications difficulties with the Science Backroom during CC and from design and performance issues with the tools (e.g. suit cameras, cuff interface, etc.) as described by Young et al. [18]. As with the design of sample bags and other tools, improvements to these technologies will require adjustments to the geologic data collection procedures.

\subsection{Metrics for mission evaluation}

The crewmembers have the responsibility to conduct investigations that achieve the science objectives while operating within the framework of the traverse/EVA plans (i.e. timeline) and the field protocols. The question then becomes: how is their science productivity defined and measured? Several metrics and numerical approaches are possible (e.g. $[22,25,26]$, in this issue), including (but not limited to) the degree to which the daily objectives are met, the incremental increase in science understanding, or a more quantitative measure such as the number of samples collected. The former two may more effectively capture the importance of geologic fieldwork, including observation, but are difficult to quantify and are subjective. The number of samples, while easily quantifiable, does not fully capture the science value of any or all samples and neglects the aspects of geologic fieldwork that are not concerned with samples.

While it is tempting to use a simple, quantitative metric like the number and weight of samples collected, our prior geological experience shows that it is very site and mission dependent. Moreover, our experience with Desert RATS has suggested to us that an overemphasis on sampling versus fundamental observation, let alone the number of samples collected, can lead to artificial pressures that result in the selection of poorly chosen samples and inadequate documentation given the strict time constraints of an EVA. Most importantly, it ignores the overriding importance of context and the value of a single "perfect" sample versus several "imperfect" ones (Section 4.1). 
Mission planners and evaluators must understand a necessary interplay between careful observations and judicious sampling and that time spent observing, as opposed to sampling, is not time wasted. Time spent making the important geologic observations and characterizing context is valuable if it results in a properly documented sample that meets the requirements of the Science Team, even if fewer samples are collected. There must not be bias towards the notion that the number of samples and the degree of geologic duplication are the most useful metrics for crew performance and science return.

\section{Recommendations}

Based on the experiences of the 2010 Desert RATS geologists, the following recommendations are offered.

\subsection{Time and task management}

To allow crews to optimally manage their time in the field, they must have as much information as possible about the entire traverse and each EVA Station in advance. This should include detailed traverse plans before even arriving at the landing site and the maximum amount of data while in the SEV. The Apollo crews, for example, were immersed in the plans, images, maps, and other data for their landing sites to the point they were intimately familiar with every detail before the mission launched. For them, this was necessary since the EVAs were so stringently designed and all but one of the astronauts were non-geologists. Armed with a similar amount of information about what is known and how the nominal mission should transpire, future planetary geologists will be well prepared to assimilate new observations obtained in the field and re-plan accordingly to maximize the science return of their traverses and EVAs.

If duplication of effort is to be avoided, it is important that the crew is always cognizant of the science objectives so that proper coordination in activities is maintained. In Desert RATS and in real missions, this can occur throughout the process of mission planning and execution through participation in the design of traverses and EVAs, as well as through Science Team briefings/debriefings and intercrew conferences. Another possibility is to pre-designate a field science lead, a geologist crewmember with oversight authority over all the field geologic activities [7]. Whatever organizational structure is adopted, it should ensure that adequate inter-crew communication occurs during the mission. To this end, crews should thoroughly summarize their activities to each other, not just the Science Team, but as the day progresses. Similarly, the end-of-day briefings should include time for the crews to talk amongst themselves in preparation for the next day of work. These communications should be in the form of verbal communications as well as all of that day's collected data, including written logs, annotated imagery, and CFNs. Most importantly in this context, some mechanism for the crews to communicate to each other during EVAs is needed because, without it, it is not possible for crews to collaborate in the flexecution process and it is not possible for one crew to be updated on exactly what the other ends up doing until it is too late.

\subsection{Field protocols}

Some modifications to the field geology protocols may help to facilitate time management while promoting the collection of better-quality observations and betterdocumented samples. Principal among these is to adjust the protocols (and EVA timeline) to include time to ensure that an outcrop is thoroughly evaluated prior to committing to a sample. The geologist can use this time to better examine and "pre-sample" an outcrop (e.g. chip off some pieces, take a close look at the mineralogy and structure, trace any lateral or vertical heterogeneities, etc.). Because the pre-sampling activities need not be formally documented (although the running commentary should be preserved), the geologist would have the opportunity to focus on the outcrop and to make a reliable first assessment before initiating the documentation process and committing the time to take a sample. In the adjusted protocol, the sample documentary process would only begin after a good sample has been identified, even potentially extracted. This may require the necessity for imagery of pristine, in-situ samples prior to sampling to be relaxed. If necessary, such imagery could, in principle, be easily reconstructed from the video archive of the EVA, provided that the crew captures a few seconds of stationary and stable video of any site that they hit with the hammer. The benefit would be improved and increased science situational awareness of the geologic materials collected. While such an assessment is natural for the terrestrial field geologist, the time constraints on EVA may pressurize even an experienced geologist into prematurely selecting a site for detailed work. Codifying this practice into the protocols may alleviate this pressure.

An increased emphasis on geologic observation will require appropriate procedures and EVA timelines. The established protocols are a useful starting point, although one elaboration already adopted by the 2010 crews was an additional mental checklist of what geologic information is vital to include in CFNs for sample documentation to ensure concise and complete notes (Fig. 1). This was necessitated by the relatively short (ca. $3 \mathrm{~min}$ ) length of the CFN. To go beyond this, additional enabling technologies are needed in order to capture the same level of observations that terrestrial geologists do, for example geologic map data [18].

Finally, in order to facilitate flexecution, the field protocols should incorporate flexibility with the understanding that the crew has been trained to recognize stops that require improvisation and that mission success may hinge on crucial contextual observations rather than on samples alone. By placing the final decision making authority for field activities on the crew, inefficiencies in fieldwork (e.g. unnecessary EVAs or samples, etc.) can be eliminated and the crew can decide what it will take to do the best science and meet mission objectives successfully. At the same time, this will increase the expectations placed upon the crew for them to pursue the correct course of action, a strong argument for the inclusion of 
field geologists on planetary exploration crews. This is also a strong argument for including a maximum amount of geologic training for all crewmembers on a planetary mission and for continual engagement in mission simulations such as Desert RATS.

\section{Field geologic concepts for future mission scenarios}

For future analog tests and real mission scenarios, two field geologic concepts will need to be explored in more detail: the role of work done while IVA and using a robotic arm; and strategies for geologic mapping on the moon (or other planetary surfaces). While neither of these was a specific focus of Desert RATS 2010 - because of a lack of a robotic arm on the SEV in the former case and because the data collection infrastructure to support mapping during EVA is not yet present in the latter case - the 2010 crewmembers have insights and suggestions regarding these issues stemming from our experiences.

\subsection{IVA operations}

During Desert RATS 2010, and in all previous field tests with the SEVs, observations of the surroundings made by IV crewmembers have been essential. As part of the nominal procedure when arriving at an outcrop, context observations are done throughout the approach to and final selection of an EVA Station (see Section 3.1). Yet, even outside the context of an EVA Station, observations from within the vehicle are important. The SEV is designed with large forward windows, including a "bubble" window, all of which afford excellent visibility. The maneuvering capabilities of the SEV also allow the pilot to position the vehicle very close to outcrops, and, using bubble to take photographs and make meso- to micro-scale observations, many observational tasks that can be performed during EVA can also be done while inside [2]. This can prove particularly valuable for saving mission time, by either omitting EVAs at Stations where IVA observations suffice to fulfill science objectives, or by allowing the collection of science observations at unplanned Stations where adding an EVA would be time-prohibitive.

Although the SEV architecture tested in 2010 did not include a robotic arm that would allow an IV crewmember to collect samples or manipulate tools outside the vehicle, future versions are likely to include such devices. These capabilities could be transformative in the flexibility afforded to exploration crews. For example, having an arm could allow samples to be collected without necessarily going EVA, allowing EVA time to be saved [27] and/or allowing an IVA crewmember to assist EVA crewmembers with their sampling and observation tasks. Use of a robotic arm will need to be evaluated in an operational sense during future Desert RATS tests, particularly how its use would interface with the other activities and procedures done on EVA.

\subsection{Mapping}

One of the most important aspects of terrestrial field geology is geologic mapping. Traditional methods will need to be adapted for planetary field geology because of the drastically different logistical and operational constraints geologist-astronauts will experience on the surface of the Moon or another planetary body. For example traditional pencil-and-paper maps and the newest digital maps made using GIS software (e.g. [28]) rely on the user drawing and writing on a map using a pencil or stylus, so they are poorly-suited for work with gloved hands in a spacesuit. Improvements to current digital mapping methods that allow for alternative modes of capturing geospatial data (e.g. voice commands, speech-to-text, and haptic interfaces) and for displaying it (e.g. heads-up augmented reality displays) can overcome these challenges (e.g $[28,29])$, but the technology needs to be developed and tested in future analog simulations.

Technological challenges aside, geologic mapping was operationally tested to some extent in the context of Desert RATS 2010. For example, the 2010 Desert RATS crewmembers experimented with rudimentary approaches to planetary geologic mapping by adapting some of the various tools available to us (see Section 3.5.2). A more extensive demonstration of the role of geologic mapping was also done as part of the traverse-planning process $[5,6]$. It relied heavily on remotely-sensed data (i.e. GeoEye imagery in Google Earth) of the test area in an effort to replicate the type of photogeologic mapping that would be done in support of a lunar mission using image datasets such as those now being acquired by Lunar Reconnaissance Orbiter (LRO; http://lunar.gsfc.nasa.gov/). The set of detailed traverse and EVA plans that formed the basis of the resulting mission plan for Desert RATS 2010 included preliminary geologic maps of the area. These were styled after the types of maps a crew would have on an actual planetary exploration mission. Both the Science Team and the 2010 crewmembers found these documents, maps, and images invaluable for understanding the context of the planned science operations, for executing the planned activities in the field, and for making decisions regarding real-time modifications to the planned activities in response to the operational and scientific circumstances encountered during the mission (i.e. flexecution). It is certain that this sort of pre-mission mapping will be a necessary part of future missions and analog simulations, playing a central role in their success.

Traditional geologic mapping, both in the context of pre-mission reconnaissance and field operations by the astronaut-geologists themselves, will also need to be adjusted for more fundamental reasons than technological and logistical considerations. Geologic mapping is a science and art developed on Earth and is in some ways specifically adapted to the type of geology seen on Earth. Hence concepts such as outcrop, stratigraphy, and structure may need to be adjusted somewhat for them to be better applicable to the geologic circumstances likely on another planetary body. For example, most of the lunar surface is covered in a layer of regolith up to kilometers in thickness [30]. Regolith is essentially broken-up and fragmented lunar crust affected by impacts and intermixed with impact ejecta from countless impacts at all scales [30]. As a result, the regolith is very complex, as are the geometric and superpositional relationships one would use to understand the relative ages of and genetic processes responsible for its components. Hypotheses about the structure and 
stratigraphy of regolith are still being developed [e.g. 31], and geologic mapping of other planetary bodies will both rely on as well as contribute to this understanding.

In this context, the single biggest obstacle to understanding any samples that would be collected from the lunar surface is the lack of detailed information about the geologic structure of the Moon: what is the lateral and vertical heterogeneity of the lunar crust of, on scales of tenths of meters to tens of kilometers? While we have some notion about what this structure is at a coarse resolution [30], fundamentally, these are questions that can only be answered in the necessary detail with geologic mapping in the field. Furthermore, while the samples themselves could be obtained using a purely robotic mission, arguably only a human-in-the-loop geologic exploration can result in the required map data and, more importantly, real-time interpretations of that data (e.g. $[8,9])$. More specifically, reconnaissance mapping of the Moon will need to address the issue of how to recognize outcrops amenable for field investigations of these questions. This will be particularly challenging for planetary surfaces where impact events, rather than tectonic or climatic events, are the primary mechanisms by which the stratigraphic and deformational history is developed and recorded. These issues are not unique to lunar geology, and they will need to be more fully considered as we explore the Moon or any other cratered planetary body.

\section{Acknowledgements}

The authors, all crewmembers for Desert RATS 2009 and/or 2010, send a heartfelt thanks to all those individuals - in both Engineering and Science and at all the NASA centers and a host of universities - who have contributed their tireless work and immense talents to making Desert RATS a success. Without your efforts, none of this would be possible. Dr. Wendell Mendell provided funding from the Constellation Program that enabled the assembly of the world-class science team for Desert RATS. J. Hurtado acknowledges funding from a 2009 NASA Field Science Analog Testing grant, NASA Minority Research and Education Program collaborative agreement \#NNX09AV09A, and NASA Lunar Science Institute cooperative agreement \#NNA09DB33A (LPI/USRA subcontract \#02173-07), which supported his participation in the 2009 and 2010 field tests. K. Young recognizes funding from the NASA Graduate Student Researchers Program Grant \#NNX10AK72H. The Goddard Space Flight Center's Solar System Exploration Division provided J. Bleacher's and J. Rice's support for the field-test. Desert RATS supporting work and manuscript preparation funding were provided by a NASA Moon and Mars Analog Mission Activities Program grant. The authors also acknowledge the suggestions of two anonymous reviewers whose input strengthened the manuscript.

\section{References}

[1] J. Kosmo, B. Romig, A. Ross, History and evolution of the desert research and technology studies analog, Acta Astronaut., this issue.
[2] W.B. Garry, J.E. Bleacher, Field geology conducted from the Lunar Electric Rover, NASA Desert RATS 2009: strategies for human surface science operations on the Moon, in: Proceedings of the 41st Lunar and Planetary Science Conference, The Woodlands, TX, 2010, abstract \#2209.

[3] W.J. Bluethmann, Title TBD, Acta Astronaut., this issue.

[4] W.B. Garry, et al., Science operations for the 2008 NASA lunar analog field test at Black Point lava flow, Arizona, in: Proceedings of the 40th Lunar and Planetary Science Conference, The Woodlands, TX, 2009, abstract \#1649.

[5] F. Horz, G.E. Lofgren, J.E. Gruener, D.B. Eppler, J.A. Skinner Jr., C.M. Fortezzo, J.S. Graf, W.J. Bluethmann, M.A. Seibert, E.R. Bell, The traverse planning process for DRATS, Acta Astronaut., this issue.

[6] J.A. Skinner Jr., C.M. Fortezzo, Traverse planning for Desert RATS 2010 activities: strategic guidance from photogeologic mapping, Acta Astronaut., this issue.

[7] J.E. Bleacher, J.M. Hurtado Jr., K. Young, J.W. Rice Jr., W.B. Garry, The effect of different operations modes on science capabilities during the 2010 Desert-RATS test: insights from the geologist crewmembers, Acta Astronaut., doi:10.1016/j.actaastro.2011.10. 018 , this issue.

[8] K.V. Hodges, H.H. Schmitt, A new paradigm for advanced planetary field geology developed through analog experiments on Earth, in: W.B. Garry, J.E. Bleacher (Eds.), Analogs for Planetary Exploration: Geological Society of America Special Paper 483, 2011, pp. 17-31, doi:10.1130/2011.2483(02).

[9] H.H. Schmitt, A.W. Snoke, M.A. Helper, J.M. Hurtado, K.V. Hodges, J.W. Rice Jr., Motives, methods, and essential preparation for planetary field geology on the Moon and Mars, in: W.B. Garry, J.E. Bleacher (Eds.), Analogs for Planetary Exploration: Geological Society of America Special Paper 483, 2011, pp. 1-15 doi:10.1130/ 2011.2483(01).

[10] G.E. Lofgren, F. Horz, the D-RATS Science Support Room, Science support room operations during Desert RATS 2009, in: Proceedings of the 41st Lunar and Planetary Science Conference, The Woodlands, TX, 2010, abstract \#2081.

[11] E.M. Jones, K. Glover, The Apollo Lunar Surface Journal (NASA), Available from:〈http://history.nasa.gov/alsj/〉, 2010.

[12] NASA, Apollo 15 Preliminary Science Report, NASA SP-289, 1972.

[13] G. Klein, Flexecution as a paradigm for replanning, Part I, IEEE Intell. Syst. 22 (2007) 79-83.

[14] G. Klein, Flexecution, Part 2: understanding and supporting flexible execution, IEEE Intell. Syst. 22 (2007) 108-112.

[15] A. Yingst, Title TBD, Acta Astronaut., this issue.

[16] NASA, Apollo 12 Preliminary Science Report, NASA SP-235, 1970.

[17] D. Eppler and the Desert RATS Science Team (41 co-authors), Desert RATS 2010 Science operations I: operational approach to managing science return from a 14 day planetary surface mission, Acta Astronaut., this issue.

[18] K.E. Young, J.M. Hurtado Jr., J.E. Bleacher, W.B. Garry, J.A. Buffington, S.A. Bleisath, J.W. Rice Jr., Tools and technologies needed for conducting planetary field geology while on EVA: insights from the 2010 Desert RATS geologists crewmembers. Acta Astronaut., doi:10.1016/j. actaastro.2011.10.016, this issue.

[19] S.G. Love, J.E. Bleacher, Crew roles and interactions in scientific space exploration, Acta Astronaut., this issue.

[20] S.Y. Lee, D. Lees, T. Cohen, M. Allan, M. Deans, T. Morse, E. Park, T. Smith, Re-useable science tools for analog exploration missions: xGDS Web Tools, Gigapan Voyage, and VERVE, Acta Astronaut., this issue.

[21] C. Evans, M. Calaway, M. Bell, GeoLab: a habitat-based laboratory for preliminary examination of geological samples, Acta Astronaut., this issue.

[22] D.B. Eppler, D. Ming, Science operations lessons learned during the conduct of the Desert RATS 2010 test, Acta Astronaut., this issue.

[23] T. Fong, M. Bualat, M.C. Deans, B. Adams, M. Allan, M. Altobelli, X. Bouyssounouse, T. Cohen, L. Flückinger, J. Garber, E. Palmer, E. Heggy, M. Helper, K.V. Hodges, J.M. Hurtado Jr., F. Jergens, T. Kennedy, L. Kobayashi, R. Landis, P. Lee, S.Y. Lee, D. Lees, J. Lum, M. Lundy, T. Shin, T. Milam, E. Pacis, E. Park, L. Pedersen, D. Schreckenghost, T. Smith, V. To, H. Utz, D. Wheeler, D.K. Young, Robotic Follow-up for Human Exploration, American Institute of Aeronautics and Astronautics, 2010.

[24] J. Gruener,Title TBD, Acta Astronaut., this issue.

[25] A. Abercromby, M. Gernhardt, J. Jadwick, Evaluation of dual pressurized rover operations during simulated planetary surface exploration, Acta Astronaut., this issue.

[26] H. Litaker Jr., R. Howard Jr., Social network analysis and dual rover communications, Acta Astronaut., this issue. 
[27] M. Helper, P. Lee, P.M., Bualat, M.B. Adams, M. Deans, T. Fong, E. Heggy, K.V. Hodges, J.M. Hurtado Jr., K. Young, Robotic follow-up to human geological and geophysical field work: experiments at Haughton Crater, Devon Island, Canada, GSA Abstracts with Programs, October 2010 Annual Meeting, Denver, CO, 2010, abstract \#180577.

[28] T.L. Pavlis, R.P. Langford, J.M. Hurtado Jr., L.F. Serpa, Computer based data acquisition and visualization systems in field geology: results from 12 years of experimentation and future potential, Geosphere 6 (3) (2010) 275-294, doi:10.1130/GES00503.1.

[29] P. Houser, J.M. Hurtado Jr., Advanced applications of mobile computing and augmented reality for field geology, GSA Abstracts with Programs, October 2010 Annual Meeting, Denver, CO, 2010, abstract \#180524.

[30] B.L. Jollif, M.A. Wieczorek, C.K. Shearer, C.R. Neal (Eds.), New Views of the Moon, Reviews in Mineralogy and Geochemistry 60, Mineralogical Society of America, 2006.

[31] G.R. Osinski, L.L. Tornabene, R.A.F. Grieve, Impact ejecta emplacement on terrestrial planets, Earth Planet. Sci. Lett. 310 (3-4) (2011) 167-181, doi:10.1016/j.epsl.2011.08.012.

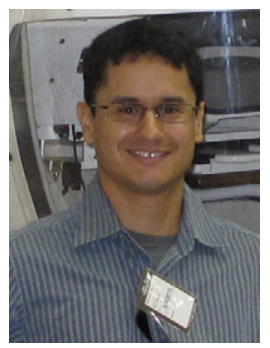

José M. Hurtado Jr. is an associate professor in the Department of Geological Sciences at the University of Texas at El Paso (UTEP) and co-investigator with the UTEP Center for Space Exploration Technology Research. His terrestrial research focuses on the tectonic evolution of the Nepal and Bhutan Himalaya using field geology, geomorphology, thermochronology, and remote sensing. His planetary science research focuses on remote sensing studies of the lunar surface to answer questions regarding in-situ resources and the geomorphology of cratered terranes. Jose served on the Desert RATS science team in 2009 and as crewmember since 2010. He earned a B.S. and M.S. in Geology from the California Institute of Technology in 1996 and a Ph.D. in Geology from the Massachusetts Institute of Technology in 2002.

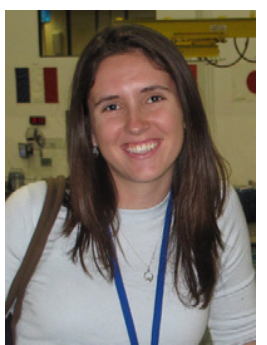

Kelsey Young is a graduate student working towards her Ph.D. in Geology in the School o Earth and Space Exploration at Arizona State University. Her research focuses on the use of terrestrial analog sites in both understanding geologic processes on other planetary bodies as well as in the context of manned space exploration. She also works on the development of handheld technology that will aid astronauts in scientific data collection in planetary field geology. Kelsey served as a Desert RATS crewmember in 2010. She earned her B.S. in Geosciences from the University of Notre Dame in 2009.

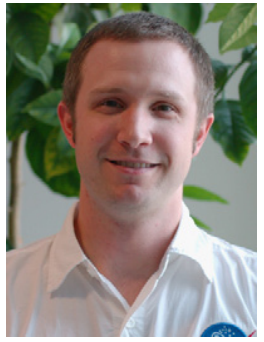

Jacob E. Bleacher is a research scientist at NASA's Goddard Space Flight Center in the Planetary Geodynamics Lab, Solar System Exploration Division. Jake's research focuses on the development and modification of planetary volcanic terrains through a combination of terrestrial field studies and spacecraft data analysis. He combines his expertise in field and planetary geology to help build and test the science capabilities of NASA's newest instrument, suit and rover technologies and has served as DRATS crew since 2009. He holds a B.A. in Geosciences from Franklin \& Marshall College and a Ph.D. in Geological Sciences from the Arizona State University.

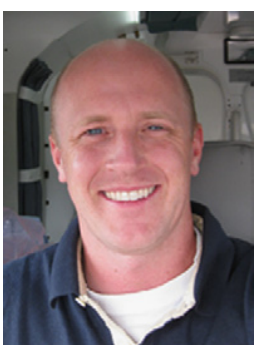

W. Brent Garry is a research scientist at the Planetary Science Institute. The focus of Dr. Garry's research is on the emplacement of lava flows on planetary bodies in the solar system. He is a Participating Scientist on two NASA missions: Lunar Reconnaissance Orbiter and Dawn. Dr. Garry served as DRATS crew in 2008 and 2009 and on the science team in 2010. He completed his Ph.D. in Geology/Volcanology at the University at Buffalo, New York (2006), and holds a B.S. in Geology from the College of William and Mary, Virginia (1999), and an M.S. in Geology from the University of Kentucky (2001).

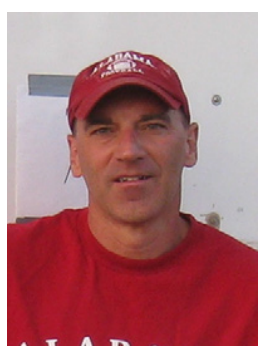

James W. Rice Jr. is an Astrogeologist working at NASA Goddard Space Flight Center. A 2010 Desert RATS crewmember, Jim is also a Science Team Member on both Mars Rove Missions (Spirit and Opportunity) and has worked on the Mars Odyssey and Mars Pola Lander Missions. His research specializes on the surface geology and history of water on Mars. Jim also conducts planetary analog field geology investigations in the Antarctic, Arctic, Iceland, Hawaii, Arizona, and California. He has been highly involved in Mars landing site selection and certification for all Mars Missions since Mars Pathfinder in 1995. Dr. Rice received his B.S. (Geology) from the University of Alabama, M.S. (Geosciences) from Northeast Louisiana University and Ph.D. from Arizona State University. 